\title{
Desarrollo y validez de un cuestionario para medir el cambio educativo en comunidades terapéuticas para drogodependientes
}

\author{
Pedrero, E.J.*; Martínez, I.M. * *; Olivar, A.*** \\ *Psicólogo. Máster en Drogodependencias. **Psicóloga. Máster en Antropología. APID. *** Educador. APID. \\ ENTIDAD: Asociación para la Prevención e Investigación en dependencias APID. \\ Enviar correspondencia a: \\ Eduardo J. Pedrero Pérez. C/ Bergantín, 11 5 B. 28042-MADRID. Teléfono: 917475872. asociacionapid@yahoo.com
}

\begin{abstract}
RESUMEN
Presentamos las etapas de elaboración y estudios de validez y fiabilidad del Cuestionario de Cambio Educativo CCE-66, diseñado para estimar los resultados de la acción educativa realizada en Comunidad Terapéutica Profesional (CTP) para drogodependientes. Basado en la Teoría de la Conducta Planificada de Ajzen, el CCE-66 pretende estimar la intención conductual de los sujetos de comportarse según determinados valores sociales, antes y después de la acción educativa en CTP. Las pruebas preliminares informan de cifras satisfactorias en cuanto a fiabilidad y validez del cuestionario, que también se ha aplicado sobre una muestra normativa de población general en la cual la variable Intención Conductual Guiada por Valores se distribuye de forma normal, lo que permite una estimación normalizada de los valores de cambio apreciados. Se ofrece el CCE-66 como instrumento válido para incluirlo en las baterías de evaluación de los equipos que trabajan en las CTP.
\end{abstract}

Palabras Clave. Comunidad Terapéutica, Evaluación, Instrumentos, Educación, Valores, Tratamiento de Drogodependientes.

\section{SUMMARY}

We present the development process and the validity and reliability studies of the 'Cuestionario de Cambio Educativo CCE-66' (Educational Change Questionnaire) aimed to estimate the results of the educational action produced by the Professional Therapeutic Community (PTC) for drug addicts. The instrument, based on the Ajzen's Theory of Planned Behavior $(T p B)$, estimates the behavioural intention of the person to behave following certain social values, before and after the educational intervention of the PTC. The preliminary analysis inform of a good reliability and validity of the questionnaire. We also have applied CCE-66 to a normative sample in which the variable 'Behavioural Intention Guided by Values' is distributed with a normal pattern, therefore it allows for a normalised estimation of the changes observed. We offer the CCE-66 as a valid instrument to be included in the evaluation battery of the teams working in the PTC.

Key words: Therapeutic Community, Evaluation, Instruments, Education, Values, Drug Addiction Treatment.

\section{INTRODUCCIÓN}

$\mathbf{L}$ as Comunidades Terapéuticas Profesionales (en adelante, CCTTPP) suelen definir su intervención en una doble dimensión: terapéutica y educativa (Comas, 1994). La primera tendría como objetivo la corrección o modificación de los factores afectivos, cognitivos o comportamentales que han colaborado a la instauración de una drogodependencia, a su mantenimiento y a la dificultad de abandonar el hábito. EI área educativa, por su parte, fijaría su objetivo en el desarrollo de las capacidades personales que posibili- tarían un adecuado ajuste entre el individuo y la sociedad a la que pertenece.

Los estudios de evaluación de Comunidad Terapéutica suelen basarse en criterios de eficiencia, por ejemplo índice de rotación, nivel de ocupación, coste plaza/día, etc. Tradicionalmente, se tiene por indicador de eficacia al índice de retención (Fernández et al, 1995), infiriendo que, al igual que sucede en otras modalidades de tratamiento (especialmente en los programas de mantenimiento con agonistas), un mayor contacto con el dispositivo favorece un mejor pronóstico (Hubbard et al. 1989). Sin embargo, y atendiendo al nivel de exigencia, las CCTTPP se sitúan 
en el extremo opuesto a los programas de mantenimiento. Su objetivo no es retener, esperando que la variable tiempo y el contacto con el dispositivo promueva cambios en los sujetos (Aguilar, 1997). Bien al contrario, en las CCTTPP el tiempo es una variable controlada (los programas se aplican en periodos preestablecidos) y el objetivo principal es promover cambios de gran calado en el comportamiento individual y social de los sujetos. En relación con esto, la capacidad de retener que muestre una Comunidad Terapéutica nos informará, si acaso, del ajuste entre el programa propuesto y la población atendida, pero no de su capacidad para promover los cambios necesarios para que el sujeto afronte en mejores condiciones su incorporación a su entorno de procedencia. La evaluación de las CCTTPP debe ser una evaluación del cambio producido a dos niveles: psicológico y educativo.

Desde perspectivas economicistas puede considerarse que la CTP es un recurso que requiere inversiones mucho más cuantiosas que otros programas, como los de mantenimiento con sustitutivos. Esta circunstancia ha supuesto la desaparición de muchas CCTTPP y pone en riesgo la subsistencia de muchas otras. Sin embargo, comparar a unos y otros programas únicamente por sus costes está absolutamente injustificado desde el punto de vista técnico y también desde el científico, y acaso sólo sea una equiparación útil desde la perspectiva política.

La CTP debe dar cuenta de los cambios psicológicos y educativos que es capaz de producir en los sujetos de la intervención y proponer la comparación con las transformaciones experimentadas en otras modalidades de tratamiento. La inclusión de estas variables justificaría la equiparación y la toma de decisiones. Sin embargo, aunque la historia de las CCTTP es ya muy extensa, no ha sido habitual la evaluación de resultados, fundamentalmente, a nuestro entender, por dos razones: por una parte, no ha sido demandada por quienes tienen la responsabilidad política de diseñar la distribución de recursos; por otra, porque las CCTTPP han sucumbido a la autocomplacencia, dando por hecho que tales resultados eran intrínsecamente buenos, más allá de su magnitud o de su existencia real.

Para la evaluación del cambio psicológico existen un buen número de instrumentos validados que pueden informarnos de la evolución de la persona en tratamiento en diferentes componentes, tanto de índole cognitivo, como comportamental o psicopatológico (Fernández et al, 1996). En el ámbito educativo, en cambio, no se han desarrollado instrumentos capaces de estimar la magnitud del cambio experimentado por un sujeto en las diversas variables educativas que se ponen en juego durante su paso por una CTP.

El objetivo de este trabajo es presentar el proceso de creación de un cuestionario capaz de medir, con criterios científicos, el cambio producido por la acción educativa en CTP.

La investigación se desarrolló por encargo de la empresa GERS S.A., gestora de la Comunidad Terapéutica Municipal de Barajas (en adelante CTB), cofinanciada por la Comunidad Autónoma de Madrid y el Ayuntamiento de Madrid (Plan Municipal contra las Drogas, 1999).

\section{LA INVESTIGACIÓN SOBRE CAMBIO EDUCATI- VOY MARCOTEÓRICO DE ESTE TRABAJO}

Una de las primeras tareas necesarias para abordar este trabajo fue la determinación del marco teórico de la CTB y del cambio educativo que se realiza con los residentes en este contexto terapéutico. Los puntos mas destacados, publicados en detalle en otro lugar (Pedrero Pérez y Martínez Higueras, 2001), fueron:

- La confluencia de los trabajos psicoterapéuticos y educativos en el desarrollo y potenciación de actividades y conductas alternativas en los drogodependientes en rehabilitación.

- La propuesta de conductas con valor tanto para el sujeto como para el entorno social de pertenencia.

- La Teoría de la Conducta Planificada de Ajzen (1988) como referente para identificar los criterios que permitirán una predicción del cambio esperado. Esta teoría tiene como eje central la consideración de que la mayor parte de la conducta humana está bajo control del propio sujeto y que, por tanto, puede ser pronosticada a partir de la intención conductual de ejecutar un determinado comportamiento, siendo la intención conductual la causa primera o inmediata de la conducta. En su formulación inicial (Fishbein y Ajzen, 1975), la intención conductual se proponía compuesta por dos dimensiones y cuatro subdimensiones:

- un factor personal o actitud: la evaluación individual positiva o negativa de llevar a cabo una acción. Tiene dos componentes:

ocreencias conductuales, convencimiento del sujeto de que realizando una conducta obtendrá unos resultados

o evaluación de resultados, valoración subjetiva de los efectos que provocará la obtención de unos resultados.

- un factor que refleja la influencia social o norma subjetiva: juicio probabilístico acerca de lo que la mayoría de los otros significativos piensan de la realización de una conducta determinada. Tiene dos componentes 
o creencias normativas: convencimiento del sujeto de lo que los otros significativos esperan que haga.

o motivación para adaptarse: disposición del sujeto a admitir las exigencias de sus otros significativos.

Con posterioridad Ajzen incluyó una nueva dimensión a considerar: control conductual percibido, definiendo este concepto como el grado de facilidad o dificultad percibido por el sujeto para realizar la conducta.

En relación al tema de valores, la escala de Rokeach (Rokeach, 1973; Rokeach y Reagan, 1980) ha sido aplicada a pacientes alcohólicos (Toler, 1975; Flores, 1985), fumadores (Kristiansen, 1985a; Kristiansen, 1985b), politoxicómanos (Toler, 1975; Miller y cols., 1973 ; 1974) y adictos a heroína por vía intravenosa (Rojo y cols. 1994), así como en trastornos psicopatológicos (Ortiz, 1985; Abad, 1989). La base teórica en la que se sustenta esta escala se explicita en el principio de que toda situación puede ser conceptualizada como un conflicto de valores, que serían creencias estables, de carácter bipolar, sobre modos personales de conducta que supondrían una elección para el individuo en base a una priorización. El conflicto se produce entre las demandas sociales y las necesidades individuales, que el individuo resolvería en su proceso de socialización a partir de las influencias externas e internas. La consecuencia sería la prioridad de determinados valores en una escala jerárquica que podría ser medida. El cuestionario de Rokeach se divide en dos mitades, correspondiendo la primera a los "valores instrumentales", que incluyen los valores morales y los valores de competencia, mientras que la segunda estimaría los "valores finalistas", que vendrían a ser la expresión de las necesidades humanas desde una perspectiva individual y motivacional.

La utilidad principal de esta escala ha sido la determinación de diferencias intergrupales, tanto en relación a variables sociodemográficas (sexo, edad, grupo étnico), como entre usuarios de diferentes drogas. Sin embargo, también se han encontrado diferencias entre los valores predominantes en grupos de pacientes en diferentes fases de tratamiento, o entre aquéllos que continúan en programa o lo abandonan. Se ha sugerido que estas diferencias podrían operar como predictores de éxito en unas u otras modalidades de tratamiento (Rojo y cols. 1994), pero no contamos con estudios que sometan a constatación esta hipótesis.

En relación al modelo de Fishbein y Ajzen, y la posterior contribución del segundo de los autores, la producción científica es muy elevada. La base de datos del PsycLIT, recoge en la última década cerca de 200 referencias a trabajos inspirados en este modelo, que han investigado la intención en relación a una multiplicidad de conductas: educación escolar, elección de carrera universitaria, conductas de jugadores, uso de cinturón de seguridad en vehículos, donación de sangre, cuidados dentales, prevención de incendios forestales, uso de preservativos, autocuidados de salud, etc. En el tema de conductas adictivas, los estudios se han centrado especialmente en el uso de tabaco (Budd, 1987; Marín et al, 1990; Sutton, 1989; Norman y Tedeschi, 1989) y de alcohol (Brubaker et al, 1987; Schlegel et al, 1987, 1992), con especial énfasis en lo relativo a la prevención. Son pocos los estudios que se aplican en el ámbito del consumo de drogas ilegales (Ajzen et al, 1982; Laflin et al, 1994).

En nuestro país pueden encontrarse estudios basados en este modelo en relación a las drogas en general (Baeza, 1992; Sánchez y Tomás, 1996; Tomás et al, 1997), al consumo de tabaco (Becoña, 1986, 1993) y de tabaco, alcohol y cannabis (Escámez, 1990; Escámez et al., 1993). De especial interés para nuestros objetivos son los dos cuestionarios recogidos en los trabajos de Becoña (1993) y de Tomás, Sánchez y García (1997) elaborados a partir de las teorías de Ajzen y Fishbein.

La mayor parte de los trabajos ponen en relación las actitudes y el consumo de sustancias. Tomás Gradolí y colaboradores (1997) abordan la construcción de un cuestionario, el CACHER, que estudia las actitudes de los sujetos en relación a su proceso de abstinencia. Navarro Botella (2000) explora, entre otras variables, las actitudes frente a las drogas y los valores sociales como factores de riesgo/protección para el consumo. Baeza (1992) utiliza el cuestionario de Escámez y Martínez para explorar las actitudes frente al consumo y el cambio experimentado tras un programa de prevención escolar. Calafat (1984) estudia el cambio actitudinal y la integración de valores para estimar la eficacia de un programa de prevención escolar. Pons, Berjano y García (1996) y Pons y Berjano (1999) exploran la relación de diversas variables psicosociales, entre las que se hallan las actitudes (medidas según la escala ACTICOL-92) y los valores (medidas con el VAL-89), con el consumo abusivo de alcohol en adolescentes. Luengo y colaboradores (1999) estudian el papel del cambio actitudinal hacia el consumo de sustancias dentro de un programa de prevención de la drogodependencia y de la conducta antisocial, utilizando el cuestionario de Escámez (1990).

No hemos encontrado, sin embargo, ninguna investigación que aborde la evaluación de programas de tratamiento en función del cambio actitudinal, cual es el propósito de nuestro trabajo, que no pretende estudiar el cambio de actitudes en relación al uso y consecuencias del uso de drogas, sino al impacto de un programa educativo sobre la intención de desarrollar conductas normalizadas.

En el marco de la "literatura gris" aparecen diversos intentos de medir las actitudes dentro de programas de tratamiento, y, en concreto, en Comunidad Tera- 
péutica, pero, como ya advertían Alonso y colaboradores (1995) "se observa una escasez de trabajos que aborden directamente el tema de los valores y su relación con el consumo de drogas". Romero y colaboradores (1999) estudian las conductas antisociales y concluyen que los sujetos que las desarrollan muestran un sistema de valores particular, una "subcultura", que orienta su conducta hacia la transgresión normativa; los individuos con conductas problema aprecian en mayor medida valores con una relevancia personal inmediata (placer, tiempo libre, sexo) y en menor medida aspectos con trascendencia social a largo plazo (justicia, solidaridad) (Luengo, 1985). Cuando el individuo no interioriza los valores en uso, su tendencia a la transgresión se manifiesta sin inhibiciones, encontrándose que la conducta problema se relaciona con un menor aprecio por valores ligados a la socialización (Romero, 1996).

A partir de los supuestos asumidos, este trabajo se propone medir la intención conductual en relación a determinados valores, antes y después de que se haya producido la intervención educativa en la CTB.

\section{MATERIAL Y MÉTODOS}

\subsection{Fases del proceso de construcción del instru- mento.}

De forma global, las etapas que nos llevaron al instrumento de evaluación del cambio educativo que íbamos buscando fueron:

1. Revisión de documentación sobre los fundamentos y bases de la CTB y de la literatura disponible sobre el cambio educativo en Comunidad Terapéutica.

2. Sesiones de trabajo con los técnicos de la CTB para identificar conjuntamente los valores y la batería inicial de ítems potencialmente medidores de éstos.

3. Depuración de los ítems iniciales del instrumento mediante análisis estadístico.

4. Elaboración de una versión piloto del cuestionario y ensayo de su aplicabilidad.

5. Estudio psicométrico de la versión piloto y final para comprobar su fiabilidad y su validez.

\subsection{Identificación de valores guía de las conductas buscada.}

Junto con los educadores de la CTB se realizaron varias sesiones de trabajo para determinar qué valores, objeto del trabajo educativo en la CTB, serían seleccionados como relevantes para la evaluación del cambio educativo producido por la intervención.
El proceso llevó finalmente a identificar 6 valores básicos sobre los que se centraría el trabajo de selección de ítems para el cuestionario. Estos valores son:

- Respeto a sí mismo

- Respeto a los otros

- Respeto al entorno físico

- Autonomía personal

- Cooperación

- Sentido de pertenencia

Cada valor (figura 1) se refleja en aquéllas conductas que son guiadas por él. A su vez, cada conducta puede predecirse a partir de la intención que el individuo manifiesta de realizarla. Los cinco componentes de la intención conductual nos pueden proporcionar su magnitud en relación con cada conducta

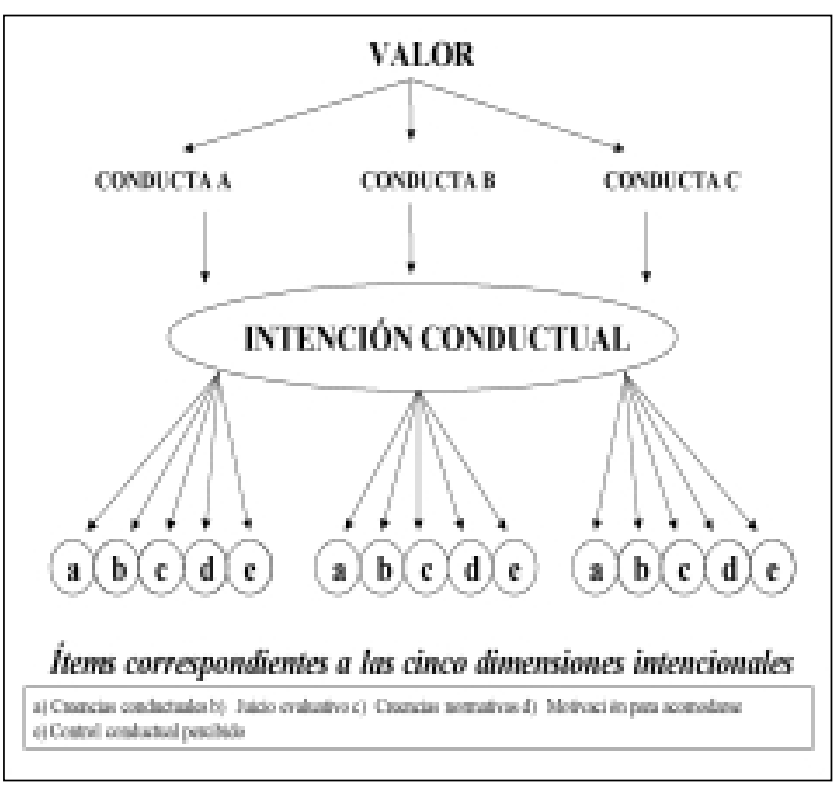

Figura 1. Representación de componentes para llegar a los ítems del cuestionario.

De acuerdo con la teoría de referencia, presentada en el capítulo 2, el sumatorio de la intención conductual (IC) de las conductas referidas a un mismo valor social (variable dependiente) puede estimarse antes y después de la intervención educativa (variable independiente).

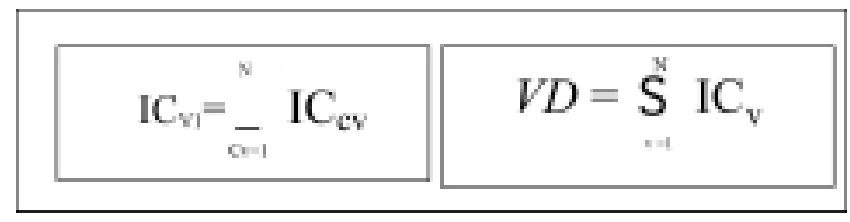

Figura 2. Formulación de la variable intención conductual guiada por valores. 
Donde la intención de comportarse según un valor social determinado (ICV1) se obtendrá a partir de la suma de cada uno de los componentes intencionales (ICcv); a su vez, la variable dependiente (que hemos denominado intención conductual guiada por valores) se obtendría al sumar el resultado de todas las conductas que se suponen guiadas por los valores predeterminados (ICV). Esta formulación se encuentra explicitada en el propio modelo propuesto por los autores (Ajzen, 1988).

Por cada valor determinado, y definiendo tres conductas relativas a cada valor, serán necesarios 15 ítems. Estimamos que 6 valores con tres conductas asociadas (90 ítems) sería una cantidad idónea para elaborar un cuestionario preliminar, breve y asequible, capaz de medir adecuadamente el cambio producido.

El método de administración sería test-retest, produciéndose el primer pase en el momento de la admisión a la CTB y el segundo en el momento del alta, cuando deberían comprobarse los cambios producidos y la magnitud de éstos.

En sesión con los educadores, y mediante trabajo por grupos, se elaboró un listado exhaustivo de conductas que pudieran estar guiadas por estos valores o sus opuestos, objeto del trabajo educativo en la CTB. Se atendió también a las Memorias anuales elaboradas desde el dispositivo (desde 1991 hasta 1998). Se procuró la agrupación de conductas específicas en grupos de conductas más generales sin perder cierto grado de especificidad, tal y como recomiendan los autores del modelo (Fishbein y Ajzen, 1980), en la línea de lo que denominan categorías conductuales, en oposición, o en el extremo de un continuo, en cuyo polo de especificidad se situarían las acciones simples. La pérdida de especificidad, según estos autores, acarrea necesariamente un decremento en la capacidad predictiva de la intención conductual, aunque, dadas las características del estudio que abordamos, las conductas objeto de evaluación deben mostrar un cierto grado de generalidad, si queremos una evaluación de la intención conductual en función de valores que las guían.

En base a este listado inicial se realizó la primera formulación de ítems del cuestionario, ítems relativos a la descomposición en elementos de la intención de manifestar dichas conductas, como refleja el esquema de la figura 1. Para ello, mediante criterio interjueces (grupo compuesto por educadores de la CT y los autores de este trabajo) se formularon preguntas que atendieran a las conductas (o categorías conductuales) seleccionadas, desde las perspectivas actitudinales y normativas y sus subcomponentes, una mitad en la dirección que indicaba cada valor y otra en la dirección opuesta. Se atendió también al banco de datos sobre instrumentos de medida en problemas de drogas EMCDDA (Morgan, 1997) y a los cuestionarios similares anteriormente elaborados por otros autores, previamente encontrados en la revisión bibliográfica, así como a las normas generales estimadas como más relevantes (Barbero, 1993).

\subsection{Selección de ítems para la batería inicial y pri- mera prueba de validez.}

Se preparó una batería de 180 ítems, 90 formulados en positivo y 90 en negativo. La batería se administró a una muestra compuesta por:

- los residentes en la CTB en el momento de la aplicación ( $\mathrm{N}=27)$.

- una muestra independiente de sujetos drogodependientes en tratamiento en diversos recursos de tratamiento ambulatorios de la red asistencial de la región $(\mathrm{N}=32)$.

- una muestra de sujetos sin problema de drogodependencias captados por el sistema de bola de nieve $(\mathrm{N}=48)$.

A partir del análisis del primer ensayo se fue realizando el cribado del cuestionario hasta llegar a su forma definitiva. Para ello se estudiaron los índices de discriminación y de fiabilidad de cada ítem, descartando aquéllos que no mostraran una significación adecuada con las escalas a las que supuestamente pertenecían o a los resultados finales del test (Muñiz, 1994).

En una $2^{a}$ fase se seleccionaron los 74 ítems que mostraron una sólida capacidad de discriminación y fiabilidad, presentándose a una nueva muestra de 52 sujetos drogodependientes (en diversos centros de la Comunidad de Madrid) y 73 sujetos sin problemas de droga conocidos (de la misma Comunidad). Nuevamente se realizó estudio de los índices mencionados, llegándose a la selección de los 66 ítems que componen el cuestionario final.

En las pruebas preliminares es de destacar la baja capacidad discriminativa de los ítems referidos al componente de 'norma subjetiva' y 'control percibido', de modo que el cuestionario definitivo quedó convertido, en su mayor parte, en un cuestionario de actitudes. Algunos estudios informan de que son las actitudes al final del tratamiento la única variable con capacidad predictiva sobre la conducta posterior (Becoña, 1993).

\subsection{Sujetos}

La muestra de sujetos a los que se aplicó la batería de ítems estuvo constituida por 422 sujetos, de los cuales 78 eran residentes en la Comunidad Terapéutica de Barajas, en diferentes etapas del proceso terapéutico; 78 sujetos estaban en tratamiento en diversos centros de tratamiento ambulatorio de la Comunidad Autónoma de Madrid y 266 sujetos sin 
problemas de drogodependencia, entre 15 y 50 años, captados con el procedimiento de 'bola de nieve'.

\subsection{Análisis y procedimiento}

Para el análisis estadístico se utilizó el SPSS para Windows 8.0.1S (1998) y se aplicaron diversas pruebas para comprobar la fiabilidad: Coeficientes $\alpha$ y Rulon y el test Guttman-Flanagan para estudiar la consistencia interna de la prueba, entre otros parámetros.

La validez se ha estudiado en contraste con un criterio externo - opinión de 5 educadores de la CTB que debían estimar las necesidades educativas de los sujetos de la muestra en una escala analógica de 6 puntos - mediante análisis de correlación de puntuaciones. También se ha determinado la correlación entre el CCE y otros cuestionarios que exploran valores (VAL-89 y RVS).

\section{RESULTADOSY DISCUSIÓN}

El cuestionario definitivo, denominado Cuestionario de Cambio Educativo (CCE), consta de 66 ítems y es el resultado de la aplicación previa de una versión con 74 ítems a la muestra reflejada en el punto 3.4. de este informe.

Los 66 ítems corresponden a 33 pares de conductas con valor positivo/negativo que han de puntuarse en una escala Likert de 4 puntos. Los ítems positivos se corrigen como +2 puntos en MUY DE ACUERDO, + 1 en DE ACUERDO, - 1 EN DESACUERDO y -2 en MUY EN DESACUERDO. Los negativos se puntúan como -2 en MUY DE ACUERDO, - 1 en DE ACUERDO y así sucesivamente.

El CCE-66 presentó una consistencia interna de 0'877 mediante el coeficiente $\alpha$ de Cronbach. Su estabilidad temporal fue de 0'83 en una muestra de 65 sujetos con un intervalo test-retest de 20 días: 45 de estos sujetos se encontraban en tratamiento $(0$ '802) y 20 eran personas del grupo control de población general (0'887). En la tabla 1 aparecen los datos obtenidos durante el análisis de la consistencia y fiabilidad.

Tabla 1. Análisis de la consistencia del CCE-66 $(\mathrm{N}=422)$

\begin{tabular}{|l|r|}
\hline \multicolumn{1}{|c|}{ PRUEBA } & \\
\hline Coeficiente $\alpha$ del test & 0,877 \\
\hline Coeficiente a de los ítems positivos & 0,805 \\
\hline Coeficiente a de los ítems negativos & 0,852 \\
\hline Coeficiente de Rulon & 0,851 \\
\hline Coeficiente Guttman-Flanagan & 0,858 \\
\hline Coeficiente a de los pares de ítems & 0,871 \\
\hline Error típico de medida & 8,16 \\
\hline Error típico de estimación & 7,65 \\
\hline Consistencia test-retest & 0,831 \\
\hline
\end{tabular}

La variable estudiada ICGV (intención conductual guiada por valores), estimada como la puntuación total del test, se distribuye de forma ajustada a la normalidad, según la prueba de Lillieford, con el $99 \%$ de nivel de confianza. La tabla 2 muestra los datos obtenidos en el análisis de la variable ICGV. Las diferencias que se observaron con el grupo no drogodependiente fueron estadísticamente significativas, estimada mediante el estadístico t de Student $(\alpha=0,005, t=6,16)$.

Tabla 2. Análisis de la Intención Conductual Guiada por Valores

\begin{tabular}{|l|c|c|}
\hline ICGV & POBLACIÓN GENERAL & DROGODEPENDIENTES \\
\hline MEDIA & 56,22 & 46,87 \\
\hline DESV.TÍPICA & 22,23 & 20,05 \\
\hline VALOR MÁXIMO & 109 & 111 \\
\hline VALOR MÍNIMO & -20 & -3 \\
\hline N & 266 & 156 \\
\hline
\end{tabular}

Se estimaron también los resultados en función de las variables sexo, nivel de estudios alcanzado y edad. Se encontró que la variable ICGV no correlaciona con la edad $(r=0,05)$; en cambio, sí resultó significativo el nivel de estudios alcanzado $(r=0,481)$. En la tabla 3 figuran los resultados de la variable nivel de estudios en la población general desglosada por tramos.

Tabla 3. Puntuación en ICGV según el nivel de estudios.

\begin{tabular}{|l|c|c|c|c|}
\hline ICGV & UNIVERSITARIO & SESO/BUP/COU & $F P$ & $\begin{array}{c}\text { ESTUDIOS } \\
\text { PRIMARIOS/SIN } \\
\text { ESTUDIOS }\end{array}$ \\
\hline Media & 67,4 & 48,4 & 46,2 & 41,3 \\
\hline Desv. Típ. & 21,1 & 18,5 & 22,4 & 21,3 \\
\hline Varianza & 446,4 & 344,3 & 500,8 & 455,4 \\
\hline $\mathrm{N}$ & 145 & 113 & 50 & 114 \\
\hline
\end{tabular}

Efectuada la prueba de Scheffé, las diferencias resultaron significativas entre todos los grupos (Tabla 4). Este hallazgo apunta a la necesidad de atender al nivel de estudios alcanzado por los sujetos drogodependientes objeto de estudio y efectuar la comparación con la distribución de esta variable en el grupo de población del mismo nivel para realizar una correcta interpretación de los resultados desde una perspectiva nomotética.

Tabla 4. Resultados de la prueba de Scheffé para estimar las diferencias entre grupos según nivel de estudios.

\begin{tabular}{|ccc|}
\hline & & $\begin{array}{c}\text { S= 3,78 } \\
\alpha=0,01\end{array}$ \\
\hline$(A-B)=$ & 6,74 & ${ }^{*}$ \\
\hline$(A-C)=$ & 11,51 & ${ }^{*}$ \\
\hline$(A-D)=$ & 6,70 & ${ }^{*}$ \\
\hline$(B-C)=$ & 12,34 & ${ }^{*}$ \\
\hline$(B-D)=$ & 7,54 & ${ }^{*}$ \\
\hline$(C-D)=$ & 12,31 & \\
\hline
\end{tabular}


Las diferencias entre sujetos drogodependientes y población general no resultó significativa en ninguno de los grupos diferenciados por el nivel de estudios alcanzado (prueba t, $\alpha=0,05$ ).

Tabla 5. Diferencias entre drogodependientes y no drogodependientes en función de su nivel de estudios.

\begin{tabular}{|l|c|c|c|c|c|}
\hline & ICGV & $\begin{array}{c}\text { UNIVERSI- } \\
\text { TARIOS }\end{array}$ & ESO/BUP/COU & FP & $\begin{array}{c}\text { ESTUDIOS } \\
\text { PRIMARIOS/SIN } \\
\text { ESTUDIOS }\end{array}$ \\
\hline & MEDIA & $\mathbf{6 6 , 8 0}$ & $\mathbf{4 5 , 1 1}$ & $\mathbf{5 1 , 2 2}$ & $\mathbf{4 2 , 8 1}$ \\
\hline DROGODEP & D.T. & 33,02 & 23,97 & 24,51 & 20,72 \\
\hline & $\mathrm{N}$ & 27,00 & 23,00 & 15,00 & 91,00 \\
\hline & MEDIA & $\mathbf{6 7 , 4 9}$ & $\mathbf{4 9 , 2 2}$ & $\mathbf{4 1 , 3 5}$ & $\mathbf{3 5 , 3 0}$ \\
\hline NO DROGODEP & D.T. & 19,49 & 16,96 & 18,80 & 19,49 \\
\hline & $\mathrm{N}$ & 130 & 90 & 23 & 23 \\
\hline & $\mathrm{t}=$ & $\mathbf{- 0 , 1 5}$ & $\mathbf{- 0 , 9 4}$ & $\mathbf{- 1 , 3 6}$ & $\mathbf{1 , 5 6}$ \\
\hline
\end{tabular}

Es preciso tener en cuenta que todos los drogodependientes que figuran en este estudio se encontraban, en el momento de la administración del test, en distintas fases de aplicación de la intervención educativa que se pretende medir (sin excluir que quienes se encontraban en tratamiento ambulatorio estén, también, participando de la acción educativa en sus correspondientes recursos), por lo cual puede mantenerse la hipótesis de que, si la estimación inicial se realizara antes de la intervención, las diferencias serían mayores y estadísticamente significativas. Dada la importancia de estos datos (puesto que, de confirmarse la inexistencia de diferencias quedaría invalidada incluso la necesidad de una intervención educativa, o bien, y más probable, la adecuación del cuestionario para estimarla) se procedió a seleccionar una muestra de 40 sujetos (10 por grupo de nivel académico alcanzado) que iniciaban tratamiento (es decir, sin haberse producido aún la intervención educativa a evaluar) y se aplicaron los mismos estadísticos, con los resultados que se muestran en la Tabla 6.

Tabla 6. Diferencias entre drogodependientes que inician tratamiento en C.T. y no drogodependientes en función de su nivel de estudios.

\begin{tabular}{|l|c|c|c|c|c|}
\hline & ICGV & $\begin{array}{c}\text { UNIVERSI- } \\
\text { TARIOS }\end{array}$ & ESO/BUP/COU & FP & $\begin{array}{c}\text { ESTUDIOS } \\
\text { PRIMARIOS/SIN } \\
\text { ESTUDIOS }\end{array}$ \\
\hline & MEDIA & $\mathbf{4 9 , 3 0}$ & $\mathbf{3 7 , 2 0}$ & $\mathbf{3 1 , 0 0}$ & $\mathbf{1 8 , 4 0}$ \\
\hline DROGODEP & D.T. & 22,27 & 24,01 & 22,76 & 16,12 \\
\hline & $\mathrm{N}$ & 10 & 10 & 10 & 10 \\
\hline & MEDIA & $\mathbf{6 7 , 4 9}$ & $\mathbf{4 9 , 2 2}$ & $\mathbf{4 1 , 3 5}$ & $\mathbf{3 5 , 3 0}$ \\
\hline NO DROGODEP & D.T. & 19,49 & 16,96 & 18,80 & 19,49 \\
\hline & $\mathrm{N}$ & 130 & 90 & 23 & 23 \\
\hline & $\mathrm{t}=$ & $\mathbf{- 2 , 7 9}$ & $\mathbf{- 2 , 0 1}$ & $\mathbf{- 1 , 3 2}$ & $\mathbf{- 2 , 3 3}$ \\
\hline
\end{tabular}

Las diferencias en este caso sí mostraban significación estadística $(\alpha=0,05)$ en tres de los cuatro gru- pos (salvo en el grupo de Formación Profesional), lo que permite mantener la hipótesis de que existen diferencias significativas en la variable estudiada que pueden ser objeto de una acción educativa, cuya efectividad puede ser estimada mediante el instrumento presentado.

Las diferencias en la variable sexo no son relevantes pues, aunque las mujeres obtenían consistentemente puntuaciones medias ligeramente superiores en todos los grupos, éstas no presentaron significación estadística, ni en la muestra general (prueba t, $\alpha=0,05)$, ni cuando se controlaba la variable nivel de estudios y la pertenencia al grupo de drogodependientes o no drogodependientes (prueba de Scheffé).

Dado que la muestra de población general se encontraba descompensada hacia los niveles superiores académicos, se realizó una equiparación a la distribución de esta variable en la población general de Madrid, mediante consulta a los Anuarios Estadísticos del Ayuntamiento de Madrid. Una vez conocida la distribución de la variable en la población general, se estimó, a partir de los datos de la muestra disponible, la media y desviación típica en cada uno de los grupos, así como en la población total. Aunque la muestra es insuficiente, puede tomarse como referencia provisional una distribución normal de la variable ICGV con media 50 y desviación típica 20. No obstante, este dato no pasa de ser, en la actualidad, un dato tentativo, en la medida en que los Anuarios consultados no se encuentran actualizados (1997) y nuestra muestra no es representativa: por encima de 1.500 sujetos podría realizarse una estimación con un nivel de confianza del 95\%.

En relación a los 6 valores propuestos, estudiamos la distribución que éstos presentaban en la muestra de población de drogodependientes y no drogodependientes. Aunque cada ítem se formuló atendiendo a un valor social que supuestamente guiaba la creencia o conducta estudiada, el análisis posterior realizado mediante criterio interjueces (en nuestro caso, los propios educadores de la CTB), puso de manifiesto que algunos ítems podían adscribirse a dos o más valores. La estructura del test quedaba según los siguientes pares:

Tabla 7. Pares de ítems adscritos a cada valor

\begin{tabular}{|l|c|}
\hline VALOR & ITEM \\
\hline Valor 1 - RESPETO POR UNO MISMO & $(3,45)(4,38)(7,53)(11,54)$ \\
& $(23,44)(32,66)(47,63)$ \\
\hline Valor 2 - RESPETO POR LOS DEMÁS & $(8,49)(12,42)(13,40)(15,50)$ \\
& $(22,37)(25,52)(32,66)(34,58)$ \\
\hline Valor 3 - RESPETO POR EL ENTORNO & $(16,41)(25,52)$ \\
& $(34,58)$ \\
\hline Valor 4 - COOPERATIVIDAD & $(1,43)(2,17)(10,48)(14,21)(22,37)$ \\
& $(30,55)(29,59)(31,36)(24,39)$ \\
\hline Valor 5 - AUTONOMÍA PERSONAL & $(5,62)(61,65)(33,64)(19,57)(8,49)(6,20)$ \\
& $(26,51)(7,53)(9,56)(25,52)(27,46)(35,28)$ \\
\hline Valor 6 - SENTIDO DE PERTENENCIA & $(4,38)(25,52)(6,20)$ \\
& $(33,64)(18,60)(34,58)$ \\
\hline
\end{tabular}


Con posterioridad, al alcanzar la muestra actual $(\mathrm{N}=422)$ procedimos a realizar un análisis factorial exploratorio (SPSS, 1999; Ferranz, 1994), que nos indicó la existencia de 4 factores y 4 pares de items marginales que no mostraban correlación significativa ni entre ellos ni con los 4 factores. Esta estructura per- mitía adscribir cada item a un solo valor, permitiendo la independencia de las escalas (Tabla 8).

El coeficiente a del test, estimado a partir de los pares de ítems, fue de 0,870. y el coeficiente de Gutman-Flanagan 0,811. El error típico de medida fue 8,02 y el de estimación 7,48.

Tabla 8. Composición de las escalas independientes del test factorizado y características psicométricas.

\begin{tabular}{|c|c|c|c|c|c|}
\hline & RESPETO & COOPERATIVIDAD & AUTONOMÍA & PERTENENCIA & MARGINALES \\
\hline \multirow[t]{10}{*}{ PARES } & 8 y 49 & 1 y 43 & 5 y 62 & 3 y 45 & 11 y 54 \\
\hline & 12 y 42 & 2 y 24 & 7 y 53 & 4 y 38 & 13 y 40 \\
\hline & 15 y 50 & 10 y 48 & 9 y 56 & 6 y 20 & 23 y 44 \\
\hline & 16 y 41 & 14 y 21 & 19 y 57 & 33 y 64 & 61 y 65 \\
\hline & 18 y 60 & 17 y 39 & 26 y 51 & 47 y 63 & \\
\hline & 22 y 37 & 29 y 59 & 28 y 35 & & \\
\hline & 25 y 52 & 30 y 55 & & & \\
\hline & 27 y 46 & 31 y 36 & & & \\
\hline & 32 y 66 & & & & \\
\hline & 34 y 58 & & & & \\
\hline $\begin{array}{l}\text { Correlación media de los } \\
\text { pares con su escala } \\
\text { (excluyendo al par) }\end{array}$ & 0,419 & 0,492 & 0,352 & 0,338 & 0,203 \\
\hline $\begin{array}{l}\text { Correlación media de los } \\
\text { pares con las demás escalas }\end{array}$ & 0,249 & 0,252 & 0,206 & 0,239 & 0,210 \\
\hline $\begin{array}{l}\text { Correlación de la } \\
\text { escala con el test }\end{array}$ & 0,816 & 0,790 & 0,656 & 0,680 & 0,583 \\
\hline Coeficiente alfa de la escala & 0,760 & 0,790 & 0,650 & 0,580 & 0,370 \\
\hline $\begin{array}{l}\text { Índice de discriminación } \\
\text { medio de los pares }\end{array}$ & 0,378 & 0,433 & 0,417 & 0,354 & 0,332 \\
\hline $\begin{array}{l}\text { Índice de fiabilidad } \\
\text { medio de los pares }\end{array}$ & 0,511 & 0,656 & 0,620 & 0,572 & 0,508 \\
\hline
\end{tabular}

En la tabla 9 se presentan los resultados del análisis de significación de las diferencias de medias, controlando la variable nivel de estudios alcanzado. En negrita se resaltan aquellas que presentan significación estadística.

Tabla 9. Estadístico $t$ de las diferencias de medias entre los drogodependientes y no drogodependientes en las escalas de valores según grupos de nivel de estudios alcanzado.

\begin{tabular}{|lccccccc|}
\hline & & Respeto & Cooperatividad & Autonomía & Pertenencia & Marginales \\
\hline UNIVERSITARIOS & $t_{0}$ & $-0,95$ & $-2,10$ & $-1,23$ & $-3,49$ & $-0,51$ \\
\hline BUP/COU & $t_{t}$ & 1,29 & 1,29 & 1,29 & 1,29 & 1,29 \\
\hline & $t_{0}$ & $-1,58$ & $-1,93$ & $-1,23$ & $-0,99$ & 0,77 \\
\hline F.P. & $t_{t}$ & $t_{0}$ & 1,29 & 1,29 & 1,29 & 1,29 & 1,29 \\
\hline & $t_{t}$ & $-1,24$ & $-1,30$ & $-1,32$ & $-1,29$ & $-1,25$ \\
\hline PRIMARIOS/ & $t_{0}$ & 1,31 & 1,31 & 1,31 & 1,31 & 1,31 \\
\hline SIN EST & $t_{t}$ & $-2,60$ & $-1,12$ & $-1,91$ & $-1,44$ & $-0,19$ \\
\hline
\end{tabular}

( $\mathrm{t}_{\circ}=\mathrm{t}$ observada; $\mathrm{t}=\mathrm{t}$ teórica para un $\alpha=0,1$ ) 
Las diferencias son significativas o se aproximan a la significación en casi todos los casos, salvo en la escala de ítems marginales.

La validez de contenido viene avalada por el propio proceso de elaboración del cuestionario, dado que son los propios educadores de la CTB los que determinan y seleccionan los aspectos más importantes a evaluar, así como las Memorias anuales del Centro, donde están reflejados estos contenidos de forma exhaustiva y explícita.

Para estimar la validez concurrente del cuestionario se extrajo una submuestra aleatoria de 21 sujetos drogodependientes y se solicitó a cinco profesionales del equipo técnico de la CTB que estimaran las necesidades de éstos en el ámbito educativo, en una escala analógica de 0 a 5 puntos y, posteriormente, se correlacionaron estas estimaciones con las puntuaciones obtenidas por los sujetos en el cuestionario CCE-66. La correlación obtenida fue de $-0,784$.

Podría considerarse como prueba de validez convergente la correlación entre el nivel de estudios alcanzado y la puntuación obtenida en el test, en tanto que ambas medidas suponen la medición de aspectos educativos. La puntuación obtenida en este caso fue $r=0,465$. Además, estimamos la validez convergente administrando a una muestra de 42 sujetos en tratamiento el CCE-66, el VAL-89 (Schwartz y Bilsky, 1987; versión de Molpeceres, 1991) y el Rokeach Value Survey (Rokeach, 1973; versión española de Ortiz y Abad, 1985) éste último aplicado mediante fichas sobre una plantilla piramidal para forzar la jerarquización de valores (en el vértice superior el valor más apreciado). Se estimó la correlación de las 5 escalas de valor del CCE con los ítems de ambos cuestionarios y se extrajeron aquéllos que correlacionaban significativamente $(\alpha=0,05)$. En general las correlaciones apuntaban a los resultados esperados. EI VAL presentaba como correlaciones positivas más destacadas en los ítems 5 (Libertad de acción y pensamiento), 14 (Respeto a mí mismo), 40 (Respetuoso), 35 (Abierto, tolerante) y 27 (Autoridad, derecho a mandar); y negativas con el 21 (Distanciamiento de las cosas mundanas). El RVS presentaba como correlaciones positivas más destacadas en los ítems 1 (Tener una vida confortable), 34 (Ser educado), 15 (Tener respeto a uno mismo) y 29 (Ser independiente); y negativas con el 12 (Tener seguridad nacional), 14 (Lograr la salvación) y 33 (Ser obediente).

Para estudiar la validez factorial del cuestionario trabajamos en la actualidad en la aplicación del análisis factorial mediante rotación ortogonal varimax (Morales, 2000)

\section{CONCLUSIONES}

El cuestionario CCE-66 que presentamos en este trabajo, supone una propuesta de evaluación de uno de los dos pilares fundamentales de la intervención en CTP: el educativo. Sus características psicométricas parecen corresponder a un instrumento que mide adecuadamente una variable relevante desde el punto de vista educativo. Su fiablidad, cercana a 0'88 sobre una muestra de 422 sujetos es aceptable; su consistencia temporal, en cambio, es más baja, en la medida en que la mayor parte de la muestra utilizada para su estimación se encontraba en pleno proceso de intervención educativa, que en buena lógica debía incidir en la variable considerada: cuando se estimaba sobre sujetos de población general, su valor se incrementaba notablemente.

En cuanto a la estructura del test, el análisis factorial exploratorio nos proporciona unas escalas congruentes con la formulación inicial, en la que se delimitan los ítems que saturan preferentemente pero no exclusivamente con la escala a la que pertenecen. Es perfectamente posible que un análisis más exhaustivo, como el que realizamos en la actualidad, nos proporcione evidencias sobre un factor común a todas las escalas y dos o tres subfactores relacionados con las escalas actualmente propuestas, en coherencia con la propia superposición de los valores desde el plano conceptual. Mención aparte merecen los ítems que hemos denominado marginales, que corresponden a dimensiones actitudinales muy específicas o de norma subjetiva; sin embargo, aunque su exclusión hubiera mejorado las propiedades psicométricas del test, hemos optado por conservarlos en tanto que suponen la medida de una dimensión que también es objeto de trabajo en la Comunidad Terapéutica y su valor es relevante en la puntuación total del test.

Las pruebas de validez parecen apoyar la capacidad del test para estimar características educativas. Aún cuando los cuestionarios utilizados para estudiar la validez concurrente exploran valores sociales, es preciso tener en cuenta que lo que el CCE-66 mide no es la aceptación personal de dichos valores, sino la intención de comportarse de acuerdo a ellos, por lo que la convergencia de medidas sólo puede ser parcial. No obstante, esta correlación existe en la dirección previsible, por lo que es posible concluir que la validez observada es aceptable.

En principio, el CCE -66 permitiría no sólo establecer la magnitud global del cambio experimentado (perspectiva ideográfica). La utilización de los resulta- 
dos pretratamiento, desde una perspectiva nomotética, puede orientar las prioridades de la intervención educativa, en tanto que proporciona datos sobre las conductas orientadas por valores menos sólidos, que serían aquellos subsidiarios de una acción educativa más intensa. También puede proporcionar una visión general sobre las necesidades de cada individuo y de cada población: es posible mantener la hipótesis de que los diversos patrones de consumo de las diferentes sustancias se correspondan con grados diferentes de socialización. En estos casos, el diseño individualizado de la intervención permitiría la ponderación diferencial de la intervención psicológica y la educativa y, en consecuencia, una distribución más efectiva y eficiente de los recursos.

Por otra parte, aunque el cuestionario se ha diseñado desde y para una CTP podría ser igualmente utilizado desde cualquier otro dispositivo que efectuara una acción educativa sobre sus usuarios, cual podrían ser centros de tratamiento ambulatorio (tanto en programas libres de drogas como en programas de sustitutivos), centros de día, pisos u otros dispositivos de reinserción, e incluso en medio abierto. La única condición sería admitir que los valores propuestos son objeto de intervención en el programa correspondiente, lo que, dado el grado de generalidad de tales valores, no parecería en principio muy difícil. Ello además permitiría la comparación de resultados entre diversos programas.

Sin duda, una de las cuestiones más polémicas puede ser la formulación teórica de la que parte el instrumento. Del mismo modo que en algunas CCTTPP la intervención psicológica se realiza desde perspectivas psicodinámicas, en otras desde ópticas sistémicas o en otras desde enfoques cognitivos-conductuales, la intervención educativa puede adscribirse a diversos modelos teóricos, que no han de ser necesariamente el propuesto. No obstante, la amplia generalidad en la formulación de los valores que guían la intervención en la CTP objeto del estudio, permiten suponer que cualquier otra CTP va a trabajar conductas similares aunque las estrategias sean diversas. Como quiera que el CCE-66 no tiene por objeto evaluar la intervención en sí misma, sino los efectos que ésta ha producido en sus destinatarios, parece razonable pensar que pueda ser utilizado en igualdad de condiciones por una amplia gama de CCTTPP.

No obstante, su mayor debilidad estriba en el hecho de que sólo supone una evaluación parcial de una intervención integral. El cambio educativo, y, en consecuencia, sociorrelacional, experimentado por un sujeto tras el paso por una CTP no es independiente del cambio experimentado en el plano psicológico; y no lo es en tanto que ambas intervenciones tampoco lo son ni desde su formulación teórica, ni desde su aplicación práctica. Para que la evaluación fuera completa, el CCE - 66 debería complementarse con una medida de los cambios psicológicos (cognitivos, afectivos, comportamentales) obtenidos tras la intervención y formar parte de una estrategia multifactorial de evaluación y seguimiento.

Otra dificultad sería estimar la estabilidad temporal de los cambios obtenidos, pero esa sería responsabilidad de los dispositivos que continuaran la cadena asistencial y utilizaran el CCE-66 en series de medidas en futuros análisis.

Finalmente, consideramos que la correcta aplicación del instrumento exige que la primera presentación se produzca inmediatamente antes de la incorporación a la CTP (o en el momento inicial de la acogida en este dispositivo) y el postest en los momentos inmediatamente anteriores o posteriores a la salida del centro. Trabajamos en la actualidad en una versión informatizada que permitirá la corrección automática del test e incluso su pase mediante el ordenador.

\section{BIBLIOGRAFÍA}

ABAD, MA; NIETO, J; LÓPEZ, C (1998). Estilo neurótico: perfiles empíricos de variables cognitivas. An Psiquiatría, 5 (5): 177-182.

AGUILAR, I (1997). Retención en Comunidad Terapéutica: La C.T. Profesional de Barajas. Adicciones, 9 (2), 181-194.

AJZEN, I(1988). Attitudes, personality, and behaviour. Chicago: The Dorsey Press.

AJZEN, I; TIMKO, C; WHITE, JB (1982). Self-monitoring and the attitude behavior relation. Journal of Personality and Social Psychology, 42, 426-35.

ALONSO, C; COMAS, D; MEGÍAS, E; PEDRERO, Y (1995). Los valores de los adolescentes y el consumo de drogas. Rev. Adicciones, 7 (1): 77-90.

BAEZA, MC (1992). Formación de Actitudes como alternativa a la prevención de la droga. Adicciones, 4 (3): 18396.

BARBERO GARCíA, M. I. (1993): La entrevista y los cuestionarios. En M.I. BARBERO GARCÍA: Métodos de elaboración de escalas. Psicometría II. Madrid: UNED.

BECOÑA, E (1986). La relación actitud-conducta. Santiago de Compostela: Grial.

BECOÑA, E (1993). La utilidad de la teoría de la acción razonada en la predicción de la abstinencia en el tratamiento de fumadores. Revista Española de Drogodependencias $18(1): 3-14$.

BRUBAKER, RG.; PRUE, DM; RYCHTARIK, RG (1987). Determinants of disulfiram acceptance among alcohol 
patients: A test of the theory of reasoned action. Addictive Behaviors, 12 (1): 43-51.

BUDD, RJ (1987). Predicting cigarette use: The need to incorporate measures of salience in the theory of reasoned action. Journal of Applied Social Psychology, 16 (8): 663-85.

CALAFAT, A. (1984): Eficacia de un programa de prevención sobre drogas, especialmente centrado en la "toma de decisiones" según sea desarrollado por especialistas o por profesores de alumnos. Drogalcohol, 9 (3): 147516.

COMAS, D (1994). Criterios y normas para la homologación de Comunidades Terapéuticas Profesionales para Toxicómanos. Madrid: APCTT.

ESCÁMEZ, J (1990). Drogas y Escuela: Una propuesta de prevención. Madrid: Dykinson.

ESCÁMEZ, J; FALCÓ, P; GARCÍA, R; et al (1993). Educación para la salud. Valencia: NAU Llibres.

FERNÁNDEZ, C; LLORENTE, JM; CARRÓN, J (1995). Sistema Estándar de Evaluación de la A.P.C.T.T. Barcelona: APCTT.

FERNÁNDEZ, C; LLORENTE, JM; GUTIÉRREZ, MA; NISO, M (1996). Características de personalidad de heroinómanos tratados en una comunidad terapéutica. Adicciones, 8 (1), 33-51.

FERRANZ, M. (1994): SPSS/PC+V 5.0. Instrucciones para el manejo de los módulos base. Profesional statistics, advances, categories and trends. Madrid: Dykinson.

FISHBEIN, M, .; AJZEN, I (1975). Belief, attitude, intention and behavior: An introduction to theory and research. Reading, Addison Wesley.

FISHBEIN, M,.; AJZEN, I (1980). Understanding attitude and predicting social behavior. New York: Prendice Hall.

FLORES, PJ (1985). Alcoholism treatment and the relationship of native American Cultural values to recovery International Journal of the Addictions, 20 (11-12): 1707-26.

HUBBARD, RL; MARSDEN, ME; RACHAL, JV et al (1989). Drug Abuse Treatment: A National Study of Effectiveness. North Carolina: Chapel Hill, The University of North Carolina Press, 1989.

KRISTIANSEN, CM (1985). Smoking, health behavior, and values priorities. Addict Behav, 10 (1): 41-4.

KRISTIANSEN, CM (1985). Smoking, health behavior, and values: a replication, refinement and extension. Addict Behav, 10 (3): 325-8.

LAFLIN, MT; MOORE-HIRSCHL, S; WEIS, DL; HAYES, BE (1994). Use of the theory of reasoned action to predict drug and alcohol use. International Journal of the Addictions, 29 (7): 927-40.

LUENGO, M.A. (1985): Values and personality: A survey of their relationship in the case of juvenile delinquency. Personality and Individual Differences, 6, 519-522.

LUENGO MARTÍN, M.A.; ROMEROTAMAMES, E.; GÓMEZ FRAGÜELA, j.a.; GUERRA LÓPEZ, A.; LENCE PEREIRO, M. (1999): La prevención del consumo de dro- gas y la conducta antisocial en la Escuela: Análisis y evaluación de un programa. Madrid: Universidad de Santiago de Compostela.

MARÍN, B; MARÍN, G; PÉREZ, EJ; et al (1990). Cultural differences in attitudes toward smoking: Developing messages using the theory of reasoned action. Journal of Applied Social Psychology, 20 (6, 2): 478-93.

MILLER, JS; SENSENIG, J; STOCKER, RB; CAMPBELL, R (1973). Value patterns of drug addicts a function of race and sex. International Journal of Addictions, 8 (4): 589-98.

MILLER, JS; SENSENIG, J; RAYMOND; JS (1974). Values structure as a predictor of type of discharge among residential addicts. International Journal of Addictions, 9 (1): 127-36.

MOLPECERES, M.A. (1991): Sistemas de valores, estilos de socialización y colectivismo familiar. Un estudio exploratorio de sus relaciones. Tesis de Licenciatura. Dirs: G.Musitu y A.M. Fontaine. Universitat de Valencia.

MORALES, P. (2000): Medición de actitudes en Psicología y Educación. Madrid: Universidad Pontificia de Comillas.

MORGAN, M. (1997): EMCCDA Instrument Bank. Core scales, sources and guidelines. Dublin: St. Patrick's College.

MUÑIZ, J. (1994): Teoría clásica de los tests. Madrid: Pirámide.

NAVARRO BOTELLA, J. (2000): Factores de riesgo y protección de carácter social relacionados con el consumo de drogas. Madrid: Plan Municipal contra las Drogas, Ayuntamiento de Madrid.

NORMAN, NM; TEDESCHI, JT (1989). Self-presentation, reasoned action, and adolescents' decisions to smoke cigarettes. Journal of Applied Social Psychology, 19 (7, 2): 543-58.

ORTIZ, M; ABAD, A (1985). Escala de valores en neuróticos. An Psiquiatría, 1 (2): 57-63.

PEDRERO PÉREZ E.J.; MARTíNEZ HIGUERAS I.M. Marco teórico para la evaluación de resultados en la comunidad terapéutica profesional: Confluencia de aspectos psicológicos y educativos. Adicciones, 13,3:253-261.

PLAN MUNICIPAL CONTRA LAS DROGAS. Memoria 1998. Madrid: Ayto. de Madrid, 1999.

PONS DÍEZ, J.; BERJANO PEIRATS, E.; GARCÍA PÉREZ, F. (1996) : Variables psicosociales que discriminan el consumo abusivo de alcohol en la adolescencia. Adicciones, 8 (2): 177-191.

PONS DÍEZ, J.; BERJANO PEIRATS, E. (1999): El consumo abusivo de alcohol en la adolescencia: Un modelo explicativo desde la Psicología Social. Madrid: Plan Nacional sobre Drogas.

ROKEACH, M (1973): The nature of human values. New York: Free Press.

ROKEACH, M; REAGAN, JF (1980). The role of values in the counseling situation. The personnel and guidance Journal, 576-81.

ROMERO, E. (1996): La predicción de la conducta antisocial: Un análisis de las variables de personalidad. Tesis doctoral. Universidad de Santiago de Compostela. 
ROMERO, E.; SOBRAL, J.; LUENGO, M.A. (1999): Personalidad y delincuencia. Entre la biología y la sociedad. Granada: Grupo Editorial Universitario.

SÁNCHEZ, E; TOMÁS, VM (1996). Actitudes y drogodependencias: Elaboración de un cuestionario de medida. Inf Psicol, 60: 50-8.

SCHLEGEL, RP; D'AVERNAS, JR; ZANNA, M; DITECCO, D; MANSKE, SR (1987). Predicting alcohol use in young adult males: A comparison of the Fishbein-Ajzen model and Jessors Problem Behavior theory. Drug Soc, 1 (4): 7-24.

SCHLEGEL, RP; D'AVERNAS, JR et al (1992). Problem drinking: A problem for the theory of reasoned action? Journal of Applied Social Psychology, 22 (5): 358-85.
SCHWARTZ, S.; BILSKY, W. (1987): Toward a universal psychological structure of human values. Journal of Personality and Social Psychology, 53 (3): 550-562.

SUTTON, S (1989). Smoking attitudes and behavior: Applications of Fishbein and Ajzen's theory of reasoned action to predicting and understanding smoking decisions. Univ London.

TOLER, C (1975). The personal values of alcoholics and addicts. Journal of Clinical Psychology, 31 (3): 554-57.

TOMÁS GRADOLÍ, VM; SÁNCHEZ HERVÁS, E; GARCÍA LÓPEZ, R (1997). Las actitudes y el mantenimiento de la abstinencia en personas drogodependientes. En Escámez, J ed: Prevención de la drogadicción. Valencia: Nau Llibres. 


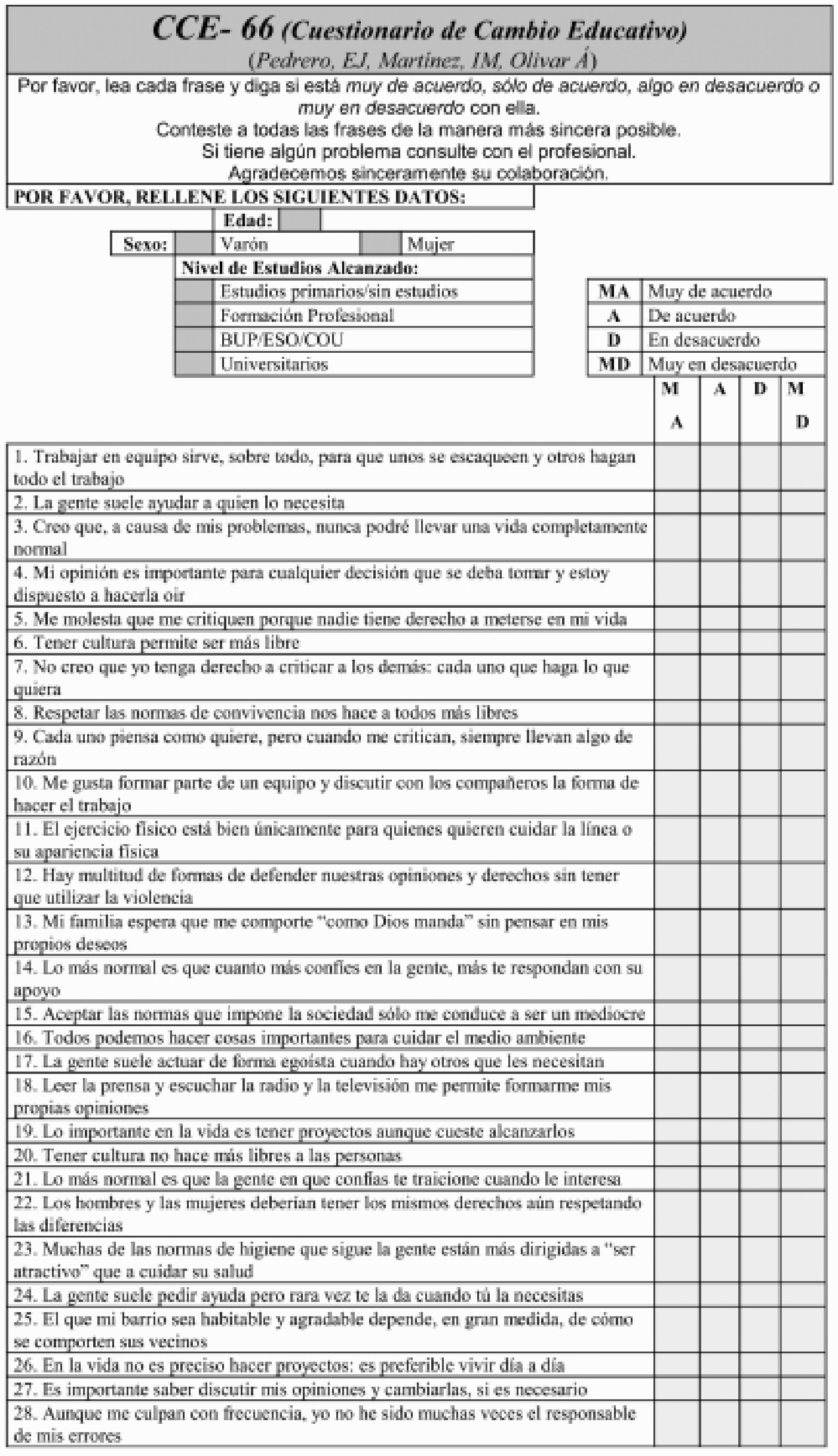




\begin{tabular}{|c|c|c|c|c|}
\hline & $\begin{array}{l}\text { M } \\
\text { A }\end{array}$ & A & D & $\begin{array}{l}\text { M } \\
\text { D }\end{array}$ \\
\hline 29. Confiar en la gente que te rodea suele traerte malas consecuencias & & & & \\
\hline 30. Según te comportes con la gente, asi se comportarán ellos contigo & & & & \\
\hline 31. Esta vida es una lucha permanente; el que no gana queda excluido & & & & \\
\hline $\begin{array}{l}\text { 32. La gente a la que aprecio (mi familia, mis amigos, mis compañeros) se } \\
\text { preocupan si hago cosas que me perjudican }\end{array}$ & & & & \\
\hline 33. En esta sociedad es imposible sentirse libre & & & & \\
\hline 34. Cuidar la limpieza de mi barrio y ciudad es responsabilidad del Ayuntamiento & & & & \\
\hline 35. Cuando algo me sale mal sé que es responsabilidad mia intentar mejorarlo & & & & \\
\hline $\begin{array}{l}\text { 36. Esta vida es una lucha permanente: cuantos más compañeros tengas en esa } \\
\text { lucha, más fácil es sobrellevarla }\end{array}$ & & & & \\
\hline $\begin{array}{l}\text { 37. No estoy de acuerdo en que hombres y mujeres deban tener los mismos } \\
\text { derechos: son diferentes y tienen funciones diferentes }\end{array}$ & & & & \\
\hline 38. En la sociedad en que vivo, nadie necesita mi opinión para tomar decisiones & & & & \\
\hline 39. He recibido ayuda de mucha gente cuando la he necesitado & & & & \\
\hline 40. Mi familia espera que, haga lo que haga, me comporte responsablemente & & & & \\
\hline 41. Cuidar el medio ambiente es inutilil la ecologia es una moda & & & & \\
\hline 42. A veces, la violencia es la única forma en que puedo defender mis derechos & & & & \\
\hline $\begin{array}{l}\text { 43. Trabajar en equipo sirve para repartir esfuerzos, para que cada uno aporte lo } \\
\text { que pueda y para que la satisfacción sea compartida }\end{array}$ & & & & \\
\hline $\begin{array}{l}\text { 44. Las normas de higiene son comportamientos necesarios para cuidar la salud y } \\
\text { prevenir la enfermedad }\end{array}$ & & & & \\
\hline 45. Soy capaz de llevar una vida tan normal como cualquier otro ciudadano & & & & \\
\hline $\begin{array}{l}\text { 46. Mi opinión es lo importante, lo que piensen los demás no me importa } \\
\text { demasiado }\end{array}$ & & & & \\
\hline $\begin{array}{l}\text { 47. Creo que soy suficientemente valioso como para merecer el respeto de los } \\
\text { demás }\end{array}$ & & & & \\
\hline 48. Prefiero siempre trabajar solo, saber lo que tengo que hacer y hacerlo & & & & \\
\hline $\begin{array}{l}\text { 49. No estoy dispuesto a comportarme como un "buen ciudadano", prefiero actuar } \\
\text { libremente }\end{array}$ & & & & \\
\hline $\begin{array}{l}\text { 50. Aceptar las normas que impone la sociedad permite que todos convivamos más } \\
\text { pacificamente }\end{array}$ & & & & \\
\hline $\begin{array}{l}\text { 51. Tener un proyecto para mi vida, saber qué quiero hacer en el futuro, me } \\
\text { resultaria de gran ayuda }\end{array}$ & & & & \\
\hline $\begin{array}{l}\text { 52. Hace tiempo que mi barrio es un lugar insoportable. La culpa es del } \\
\text { Ayuntamiento, los vecinos no podemos hacer nada }\end{array}$ & & & & \\
\hline $\begin{array}{l}\text { 53. Hacer oir mis criticas es importante para mi y para quien las recibe, porque } \\
\text { puede ayudarle a mejorar }\end{array}$ & & & & \\
\hline 54. El ejercicio fisico es importante, sobre todo, para mantener una buena salud & & & & \\
\hline 55. Hagas lo que hagas, la gente siempre va a lo suyo & & & & \\
\hline 56. Cada uno piensa como quiere y nadie tiene derecho a criticar mis opiniones & & & & \\
\hline $\begin{array}{l}\text { 57. Lo importante en la vida es vivirla intensamente cada dia sin pensar en el } \\
\text { futuro }\end{array}$ & & & & \\
\hline $\begin{array}{l}\text { 58. Yo también puedo hacer cosas que ayuden a que mi barrio o ciudad estén } \\
\text { limpios }\end{array}$ & & & & \\
\hline 59. Es posible confiar en la gente que te rodea y ver que te responde & & & & \\
\hline $\begin{array}{l}\text { 60. Generalmente, lo que dicen los periódicos no me interesa, porque suelen ser } \\
\text { mentiras }\end{array}$ & & & & \\
\hline 61. Me gustan los trabajos que duran poco y permiten ver pronto el resultado & & & & \\
\hline 62. Recibir criticas sobre lo que hago me resulta necesario para mejorar & & & & \\
\hline 63. Por regla general, nadie me ha considerado nunca como alguien importante & & & & \\
\hline 64. Soy capaz de actuar libremente la mayoria de las ocasiones & & & & \\
\hline 65. Me gusta hacer cosas complicadas, que lleven su tiempo, con tal que queden & & & & \\
\hline bien & & & & \\
\hline $\begin{array}{l}\text { 66. Hacer cosas que me perjudiquen es cosa mía y no deberia importarle a nadie } \\
\text { más }\end{array}$ & & & & \\
\hline
\end{tabular}

